\title{
ObSERVING THE OCEAN Side OF THE AIR-SEA INTERFACE
}

\author{
By David M. Farmer
}

\begin{abstract}
A face is one of the most energetic environments on the surface of the planet. The rate at which wind does work on the ocean is sensitively dependent on its speed, and effects of one severe storm can far outweigh long periods of lighter winds.

Much work has gone into establishing empirical coefficients representing airsea drag, heat exchange, and related properties, but the behavior and small scale structure of the ocean surface layer at higher wind speeds have remained largely inaccessible because of the violence it does to instrumentation and the fact that ship-based observations are limited at high sea states. Laboratory studies have been useful in identifying the relevant physics, but scaling difficulties and other limitations emphasize the need for detailed field measurements. Measurements from aircraft and satellites provide unprecedented spatial coverage but tend to be restricted to the airsea interface itself, leaving a gap in our knowledge of processes occurring just beneath the surface. In recent years this situation has begun to change as oceanographers turn to the upper ocean boundary layer with increasing interest and a variety of specialized measurement approaches.

The observational task is daunting because the scale of dominant fluid processes tends to decrease close to the surface and the surface itself can be in rapid motion (Fig. 1)! Several new measurement techniques are now being developed for studying breaking waves, surface bubble layers, turbulence, thermal struc-
\end{abstract}

David M. Farmer, Institute of Ocean Sciences, 9860 West Saanich Rd., Sidney, BC V8L 4B3, Canada. ture, and circulation patterns, even in extreme conditions. Acoustical methods play an important but by no means exclusive role in this endeavor. In this article a few recent approaches are discussed with emphasis on measurements at, and just beneath the surface, so as to illustrate ways in which they can contribute to our understanding of small scale processes at higher sea states. No attempt is made to be inclusive, and the focus is primarily on techniques with which the author, his students, and associates have been involved.

\section{Breaking Waves and Bubble Clouds}

Aside from its role in dissipating surface wave energy, wave breaking is an important source of turbulence, air entrain- ment, and momentum transfer (Thorpe, 1995; Melville, 1996); accurate measurement of its occurrence and properties is of high priority. The entrained air has a dynamic significance because it represents an exchange of kinetic for potential energy within the water column, and the bubbles almost certainly have an influence on turbulence close to the surface. Clouds of bubbles formed by wave breaking play a role in air-sea gas exchange and can serve as tracers of fluid motion. Large bubbles rise quickly, whereas smaller ones (radius $<500 \mu \mathrm{m}$ ) are redistributed by near surface turbulence and the more persistent subsurface motions we identify as Langmuir circulation. Thus the smaller bubbles are organized into patterns by the circulation.

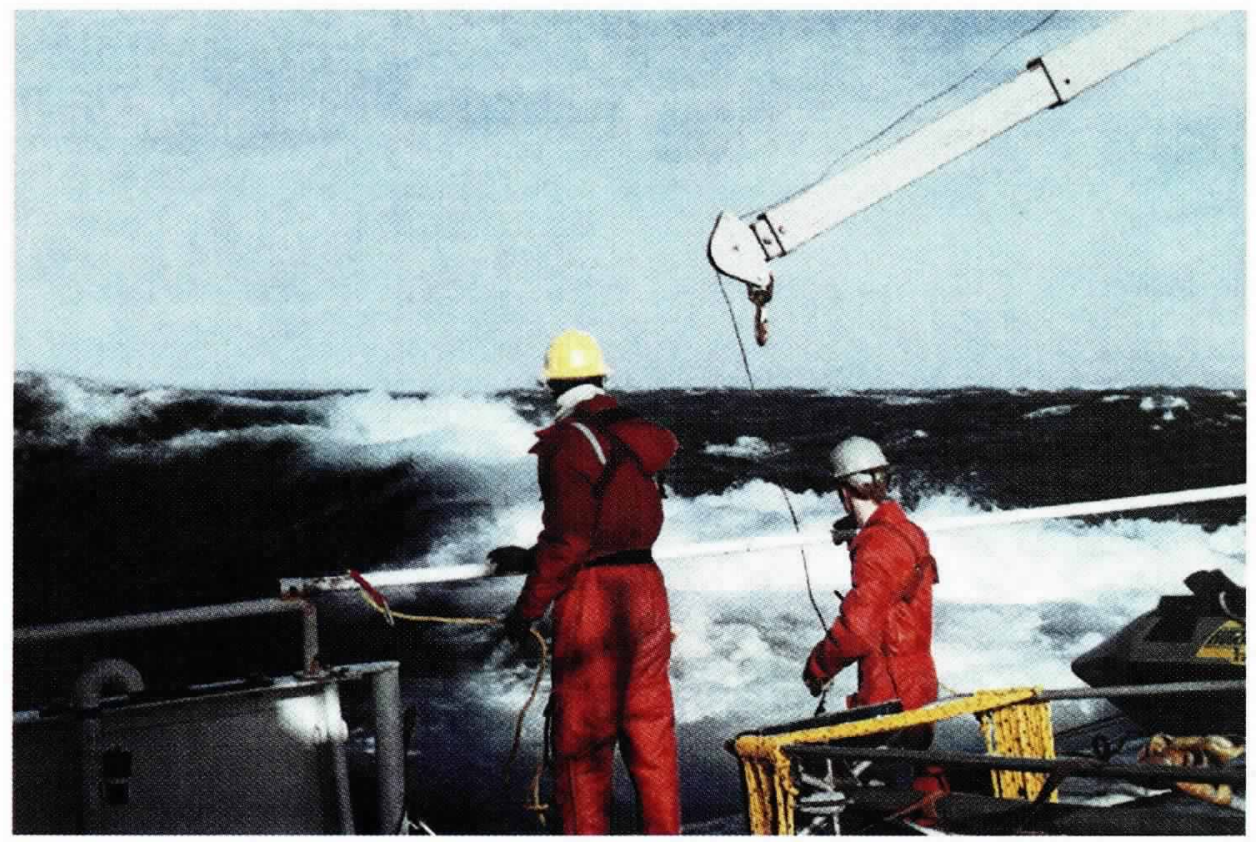

Fig. 1: Preparing to recover and redeploy a tracking buoy for monitoring neutrally buoyant floats (see Fig. 4). 
Although the study of ocean surface processes can be approached in many ways, the distribution, behavior, and consequences of near surface bubble fields provides a fairly inclusive perspective, depending as it does on surface waves and wave breaking, near surface turbulence, Langmuir circulation, and gas transfer across the bubble skin. A comprehensive model of bubble distributions must account for the factors that determine their initial size distribution and rate of production in breaking surface waves, the role of turbulence and advection in redistributing them, and the processes affecting their growth or dissolution. The rise speed of a bubble depends on its radius and the effect of contaminants on the bubble skin. The bubble radius distribution provides insight on the mechanisms associated with bubble formation, effects of buoyancy sorting, and gas exchange. Much effort is presently being invested in development of techniques for the accurate measurement of bubble size distribution. Calculation of gas dissolution from bubbles also demands improved measurement of dissolved gases.

Bubbles are effective sources and scatterers of sound (Leighton, 1994). Each bubble has a natural frequency at which it resonates, depending primarily on its radius and ambient pressure. Thermal conduction through the bubble wall absorbs some of the energy of oscillation, but this process is inefficient, giving bubbles a high "quality factor" at resonance. For example, a bubble of radius $500 \mu \mathrm{m}$ resonates at $6.7 \mathrm{kHz}$ and has an acoustic scattering cross-section of $15.4 \mathrm{~cm}^{2}$, which is $\sim 2,000$ times its geometrical cross-section. Breaking waves also generate noise; together with precipitation, they are a primary source of naturally occurring ocean sound, thus providing a useful remote sensing signal. Newly formed bubbles tend to radiate at their resonant frequency, a property that may permit determination of bubble size distributions at their point of creation.

The sound generated by breaking waves allows them to be tracked with hydrophone arrays. From the measured speed, it is possible to estimate the scale of the breaking wave components and hence the scale at which energy is being extracted from the wave field. A recent extension of this concept is the application of a hydrophone array facing a convex acoustical reflector directed toward the ocean surface (Buckingham et al.,
1996), which has been used to generate images of breaking waves in light winds. In this configuration the frequency of the sound can be considered as playing the role of color in an optical imaging system. The observations show a constantly evolving pattern of radiation at different frequencies as the wave develops, with the frequency presumably corresponding to the changing size distribution of the bubbles being formed. High air fractions can also be detected with in situ sensors. For example, electrical conductivity varies systematically with air fraction, and a conductivity sensor deployed just beneath the air-sea interface can be used to measure the occurrence of wave breaking.

Sound transmitted by sonars is scattered back to the hydrophone by bubbles having resonant frequencies close to that of the sonar (Fig. 2). Ocean surface bubble clouds have a broad size spectrum, so that sonars operating from $10 \mathrm{kHz}$ to several hundred kilohertz readily detect bubble clouds. If the sonar points directly upward, it detects bubble concentration as a function of depth and shows bubble clouds penetrating many meters downward. However, bubble densities in severe conditions can be great enough to mask the scattering signal deep within the clouds, complicating estimation of bubble concentrations by this method at high sea states. As shown in Thorpe's pioneering studies, sonars inclined away from the surface can measure the horizontal distribution of bubbles and have proved espe- cially fruitful in the study of coherent flows, such as Langmuir circulation, which form bubble cloud patterns aligned with the wind. The concept may usefully be extended either by rotating the sonar like a radar antenna or by electronically steering the beam in many different directions for each transmission (Pinkel et al., 1995; Smith and Largier, 1995) so as to generate two-dimensional images. Sequences of such images show the time evolving structure of bubble clouds collecting in convergence zones. Observations with our imaging sonar (Fig. 3) emphasize the importance of three-dimensional structures, especially at higher wind speeds and in deeper mixing layers in which Langmuir vortices interact and merge to produce complicated patterns (Farmer and Li, 1995). Observed deviations between cell orientation and wind direction result from misalignment between wind and waves (Polonichko, 1997); modulations over periods of $\sim 20$ min, possibly associated with the buoyancy torque of bubble clouds, have also been described.

Doppler processing of the bubble distribution signals provides measurements of the velocity field resolved along the direction of the sonar beam. Over short time scales (seconds) the flow speed is dominated by waves, which is useful for determining the directional wave field, but more persistent circulation patterns responsible for organizing bubble clouds can also be observed.

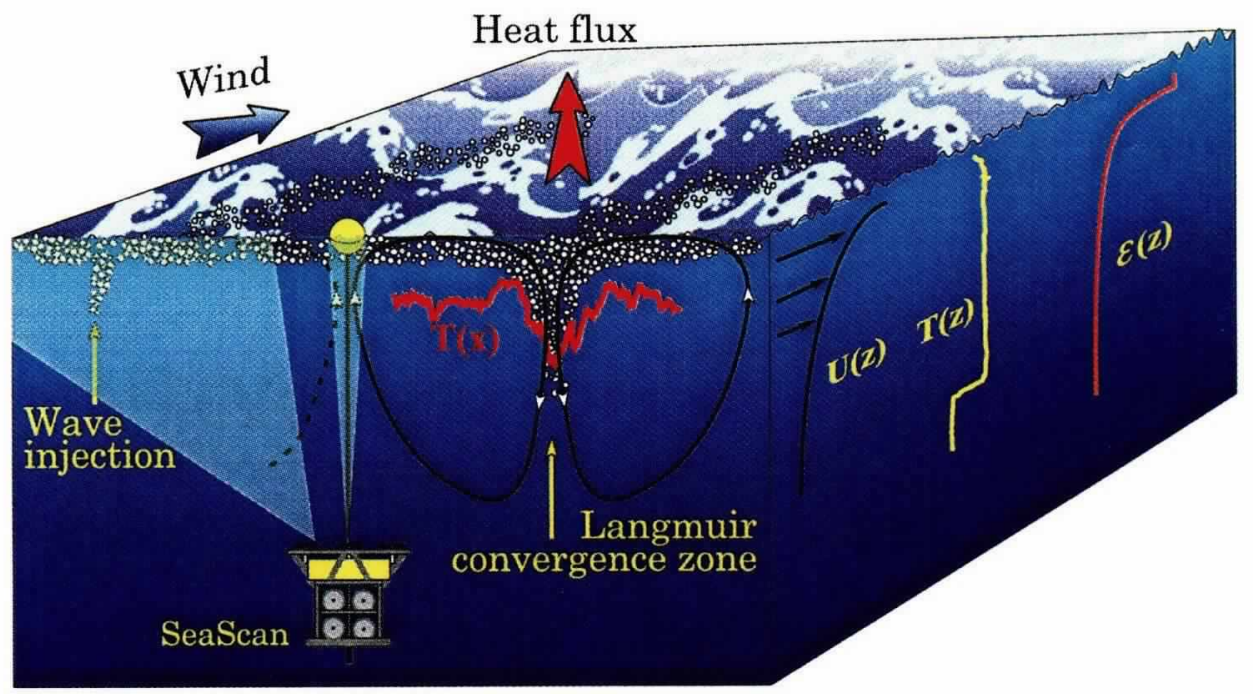

Fig. 2: Breaking waves generate bubble clouds that can be measured with sonars as they evolve under the influence of turbulence and advection. The surface heat flux produces small but measurable near surface temperature signatures $T(x, z)$. Wave breaking enhances near surface turbulence dissipation $\varepsilon(z)$. 

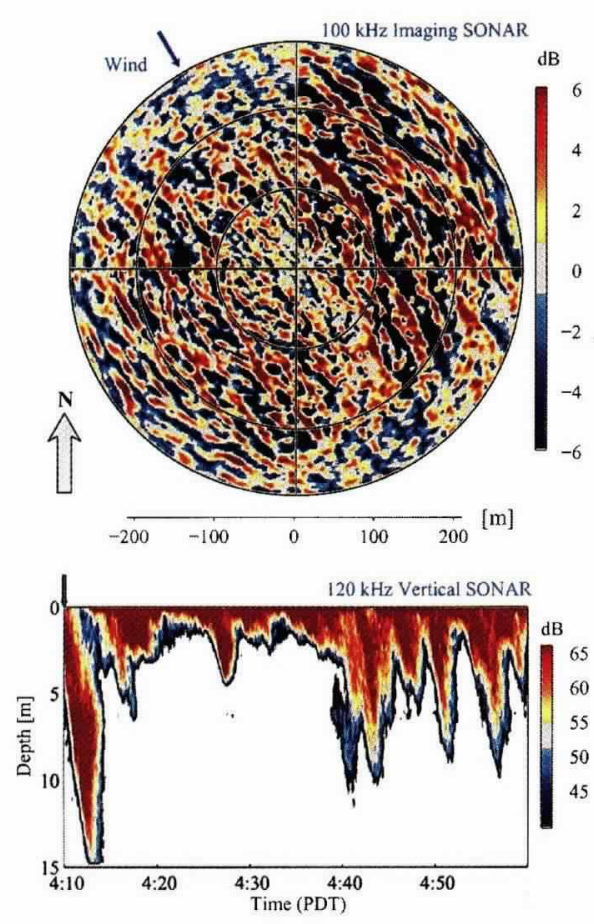

Fig. 3: (Top) Scanning sonar image made with the Institute of Ocean Sciences (IOS) Seascan sonar suspended 25 $m$ beneath the surface, showing bubble cloud patterns aligned in the wind direction (wind speed $15 \mathrm{~ms}^{-1}$ ). A $360^{\circ}$ image is obtained every $30 \mathrm{~s}$. (Bottom) Time series from upward pointing sonar signal showing bubble cloud penetration. Green arrow corresponds to time of polar image shown above. Bubble clouds are inclined, consistent with separate Doppler measurements of a $0.08-\mathrm{ms}^{-1}$ penetration speed.

The size of a bubble changes continuously as it responds to pressure changes and gas transfer through the bubble wall so that a comprehensive analysis of upper ocean bubble distributions requires measurement of bubble radii. Acoustical methods offer much promise, and there are a number of in situ "bubble spectrometers" presently under development, including both linear and nonlinear methods. The linear methods, such as resonators and travel time sonars, all depend on the fact that the bulk acoustical properties of water are modified by bubbles, whereas nonlinear methods involve an observation of bubble response to strong forcing. Vertical arrays of linear acoustical in situ bubble sensors deployed in the open ocean are now yielding measurements of size distributions that show great variability as the bubbles progress from their formation to dissolution or escape to the surface.
Acoustical methods are hard to use effectively in the high air fractions encountered within whitecaps. Optical methods appear better suited to this task, and it is likely that some combination of methods will be optimal for sampling the full range of bubble concentrations. The size distribution of bubbles generated within the whitecap must be related to the energetics of the breaking process. As we learn more about bubble formation, it may be possible to use bubble size distributions as a signal from which to infer properties of turbulence within the breaking wave itself.

Bubble dissolution depends on the difference in partial pressures of the primary gas constituents $\mathrm{N}_{2}, \mathrm{O}_{2}$ on either side of the bubble surface. Despite its relevance to the air-sea $\mathrm{CO}_{2}$ flux, the dissolution of highly soluble trace gases has no perceptible influence on bubble volume and hence buoyancy. Nevertheless, it appears that bubbles may have a significant effect on the $\mathrm{CO}_{2}$ flux (Keeling, 1993), providing added motivation for understanding dissolution of $\mathrm{N}_{2}$ and $\mathrm{O}_{2}$. Although dissolved oxygen measurements are routine for oceanographers, nitrogen is not normally measured. By determining the total gas pressure with a "Gas Tension Device" and independently measuring the dissolved oxygen, we can estimate the nitrogen partial pressure for inclusion in model analyses of acoustically derived bubble populations (Farmer et al., 1993). Nitrogen is biologically inert over short periods (days to weeks) so that the relative air-sea transfer of nitrogen and oxygen can be calculated from knowledge of their partial pressures and solubilities allowing separation of physical and biological contributions to the dissolved oxygen budget (McNeil and Farmer, 1996) with application to monitoring primary production.

\section{Lagrangian Measurement of Fluxes}

Vertically oriented Doppler sonars can detect the vertical velocity, but although current meters have been used to measure downwelling in Langmuir convergence zones (Weller and Price, 1988), separation of small vertical currents from the large signals due to wave orbital motion and instrument movement is difficult. Neutrally buoyant floats designed to follow water motions while recording depth and other properties as they move through the surface mixing layer have the potential for direct measurement of fluxes in the upper ocean (D'Asaro, et al., 1996). Many characteristics of the flow can be derived from such data, including vertical transport of properties measured with sensors on the floats. For example, changes in the temperature of the water (Fig. 4), detected at the same time as a pressure determination of vertical velocity, provide a measure of the vertical heat flux.

In collaboration with Eric D'Asaro and Geoffrey Dairiki, we have recently developed drifting surface buoys that combine Global Positioning System (GPS) satellite positioning and acoustical communication with the subsurface floats. The pressure measurements are combined with acoustic travel time and GPS positioning to develop three-dimensional tra-
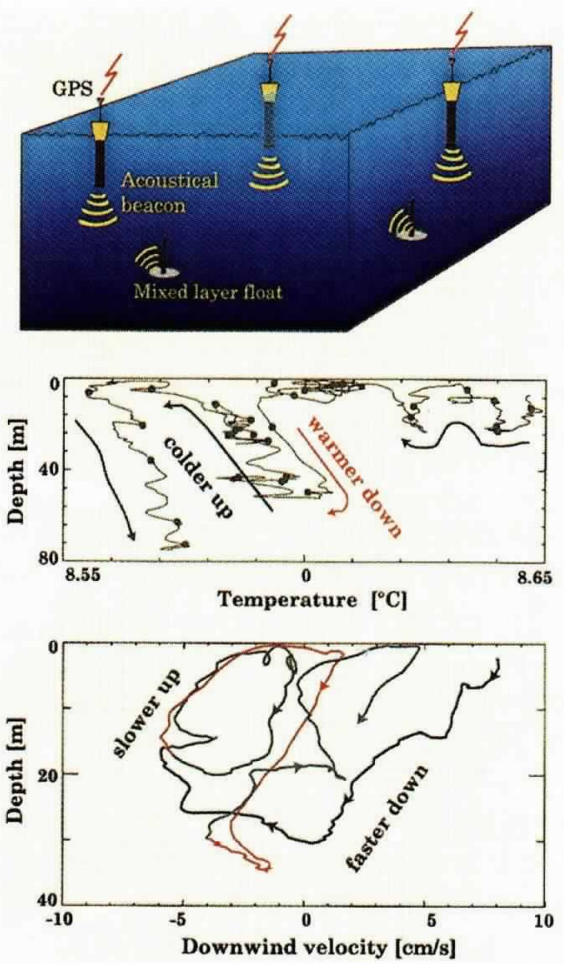

Fig. 4: (Top) Neutrally buoyant subsurface Lagrangian floats are acoustically tracked from IOS drifting surface buoys, which are themselves positioned by GPS. (Middle) Temperature-depth track showing float getting warmer as it descends, cooler as it ascends, corresponding to a positive vertical heat flux. Dots shown at 1 - $h$ intervals. (Bottom) Downwind velocity derived from acoustic/GPS positioning, against depth, showing acceleration near the surface, deceleration below. Colors change every hour. (Middle and bottom figures from Geoffrey Dairiki and Eric D'Asaro). 
jectories of the turbulent motions beneath the surface. The results (Fig. 4) show downwind acceleration of the floats close to the surface and corresponding deceleration at depth, illustrating the way in which three-dimensional positioning can be used to measure the vertical flux of momentum. Although this technology remains in a relatively early stage of development, it seems likely that Lagrangian measurements will provide an effective technique for measuring fluxes of many physical, biological, and chemical properties in the upper ocean.

\section{Turbulence Near the Ocean Surface}

Large numbers of smaller bubbles with low rise speeds (i.e., $0.2 \mathrm{~cm} / \mathrm{s}$ for a $33-\mu \mathrm{m}$ radius bubble resonant at $100 \mathrm{kHz}$ as in Fig. 3) are essentially passive within the energetic surface layer, whereas buoyancy effects are important in determining the distribution of much larger bubbles. A comprehensive understanding of near surface bubble distributions therefore requires measurement of small scale turbulence that is also of direct relevance to energy loss from the surface wave field. Near surface observations of dissipation greatly exceed "law-of-the-wall" predictions of turbulent flow past solid boundaries, a result that has also been incorporated in theoretical models. Direct measurement of turbulent dissipation at high sea states is especially difficult because of the large shears associated with wave motion. Free rising microstructure sensors have provided useful results but can only be deployed infrequently and thus far have not been used at high sea states or in conjunction with independent information on the location of the profile with respect to wave breaking, Langmuir circulation, and bubble distribution.

An indirect method of assessing near surface turbulence is through measurement of fine scale temperature structure. There is almost invariably an exchange of heat across the air-sea interface during a storm, and the formation of a $\sim 1-\mathrm{mm}$ thick molecular "cool skin" is well known to those using satellite remote sensing for ocean temperature measurement. Infrared imaging has been used to measure cool skin recovery following its disruption by wave breaking (Jessup et al., 1997). The recovery rate is related to subsurface turbulence so that infrared imagery of wave breaking may provide insight on very small scale near surface dynamics. Beneath the cool skin, we have been measuring small temperature changes in the upper two meters with a motor-driven profiling thermistor at wind speeds up to $\sim 18 \mathrm{~m} / \mathrm{s}$. The measurements are referenced to the instantaneous surface elevation with a capacitance wire gauge. Temperature profiles at scales much larger than the cool skin show slightly cooler water near the sea surface (Fig. 5), expressing the balance between surface heat flux and heat redistribution by turbulence within the water column. These results have been combined with independent knowledge of the air-sea heat flux to measure enhanced turbulence levels associated with breaking waves. Fine structure in the individual profiles yields additional insight on the turbulence scales while systematic changes in temperature can be related to Langmuir convergence zones (Gemmrich 1997).

Although the fine scale measurement of temperature is useful for inferring turbulent diffusion, it would also be helpful to measure turbulent dissipation more directly. Coherent acoustic Doppler systems operating at megahertz frequencies use a rapid sequence of closely spaced transmissions to measure velocity structure with centimeter resolution over short paths. Figure 6 shows an example of turbulence measured in this way beneath a breaking wave off Scripps Pier, which serves as a convenient testing environment for breaking wave sensors. High

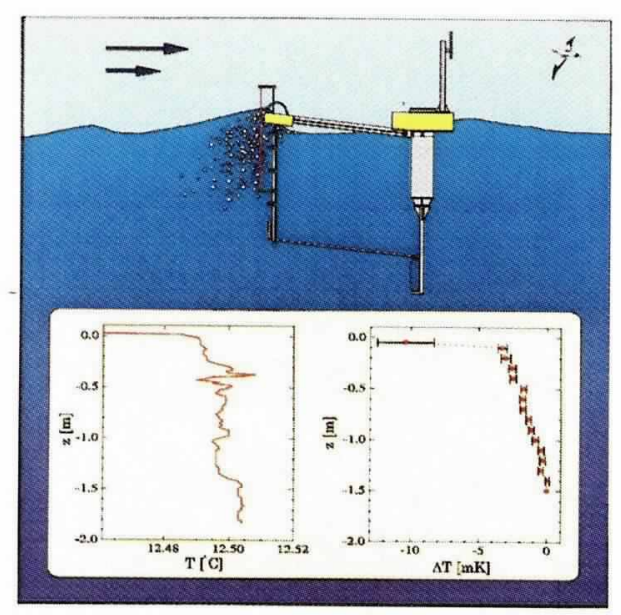

Fig. 5: Fine scale temperature profiles acquired with a motor-driven thermistor. The sensor depth is referenced to the surface by capacitance wire gauge. (Left) Individual profile. (Right) Overnight average profile. Small measured gradients together with independently known heat flux can be used to derive turbulent diffusivity.

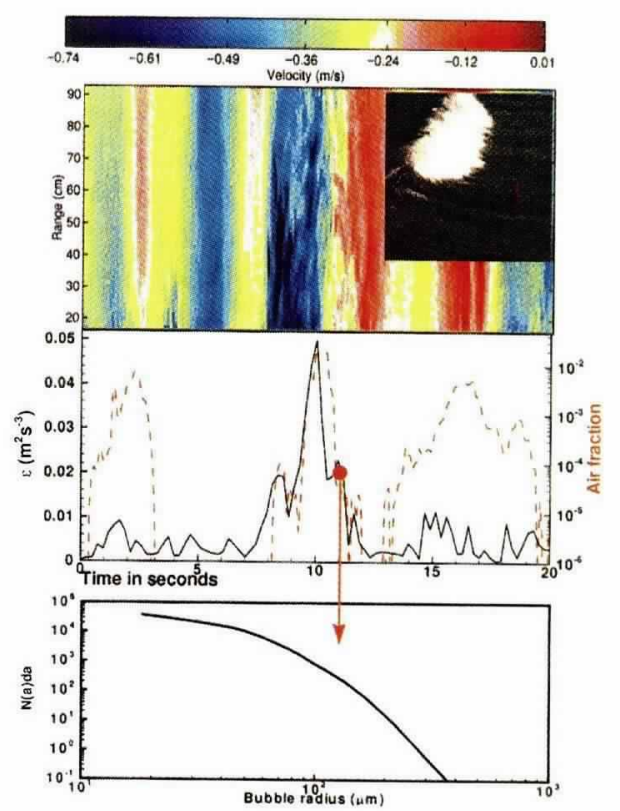

Fig. 6: Breaking wave activity off Scripps pier. (Top) Velocity fine structure measured with coherent Doppler. (Middle) Turbulence dissipation $\varepsilon$ derived from Doppler (black line); air fraction derived from acoustical resonator (red dashed line). (Bottom) Number $N(a)$ of bubbles/micrometer radius increment $/ \mathrm{m}^{3}$, against bubble radius, for the indicated sample time. Bubble measurements are made twice a second.

turbulence intensities are generated by the breaking wave and diffuse downward through the water column. The turbulent field is detected as the trough of the wave passes and the sensor moves close to the surface. Simultaneous measurements of bubble concentration show that high turbulence dissipation coincides with high air content, but the time taken for turbulence to decay is evidently much less than the time required for bubbles to dissolve or rise to the surface, so that bubble clouds are also observed without the same high levels of turbulence. Although this technology has yet to be applied to the open ocean, it is representative of the way in which the range of measurement scales in the ocean surface layer is steadily increasing. Perhaps the greatest challenge ahead lies in the interpretation of data acquired simultaneously with a broad suite of measurement approaches. This interpretation will require improved models that combine the dynamics of wave breaking, bubble formation and behavior, turbulence, Langmuir circulation, and related phenomena including antici- 
pated feedback mechanisms such as effects of bubbles on turbulence.

\section{Concluding Remarks}

No discussion of innovative measurements in the surface layer would be complete without reference to Irving Langmuir's insightful studies of Lake George in the 1930s. Armed with a few everyday items such as light bulbs and umbrellas (Langmuir, 1938), he was able to deduce several salient features of the circulation pattern that bears his name. Although most of the techniques discussed here lack the elegant simplicity (and low cost!) of Langmuir's original approach. they are providing us with previously inaccessible views of small scale structure in the ocean surface layer at higher wind speeds. In combination with thermal imaging and profiling, acoustical techniques appear particularly suitable for ocean surface studies. More comprehensive and refined measurements will provide further motivation for development of improved models that will also assist in the interpretation of multidimensional data sets. We anticipate rapid advances in our understanding of this climatically important and energetic ocean boundary.

\section{Acknowledgement}

Much of the work described here was carried out in collaboration with students, colleagues, and staff at the Institute of Ocean Sciences and with financial support from the U.S. Office of Naval Research. I am particularly indebted to S. Vagle, J. Gemmrich, and V. Polonichko for assistance with the figures.

\section{References}

Buckingham, M.J., J.R. Potter and C.L. Epifanio, 1996: Seeing underwater with background noise. Scientific American. 274, 86-90.

D'Asaro, E.A., D.M. Farmer, J. Osse and G.T. Dairiki. 1996: A Lagrangian Float, JAOTech. 13, 1230-1246.

Farmer, D. and M. Li, 1995: Patterns of bubble clouds organized by Langmuir circulation. $J$. Phys. Oceanogr., 25, 1426-1440.

Farmer, D.M. C. McNeil and B. Johnson, 1993: Evidence for the importance of bubbles to the enhancement of air-sea gas flux. Nature, $361,620-623$.

Gemmrich, J.R., 1997: A study of turbulence and fine scale temperature variability of the ocean thermal boundary layer under breaking surface waves. Ph.D. thesis, University of Victoria, Victoria BC, $209 \mathrm{pp}$.
Jessup, A.T.. C.J. Zappa. M.R. Loewen and V. Hesany, 1997: Infrared remote sensing of breaking waves. Nature, $385,52-55$.

Keeling, R.F., 1993: On the role of large bubbles in air-sea gas exchange and supersaturation in the ocean. J. Mar. Res., 51, 237-271.

Langmuir, I., 1938: Surface motion of water induced by wind. Science, 87, 119-123.

Leighton, T.G.. 1994: The Acoustic Bubble, Academic Press, Harcourt Brace \& Company, London, ISBN 0-12441920-8.

McNeil, C. and D.M. Farmer. 1996: Observations of the influence of diurnal convection on upper ocean dissolved gas measurements, $J$. Mar. Res., 53, 151-169.

Melville, W.K. 1996: The role of surface-wave breaking in air-sea interaction. Annu. Rev. Fluid Mech., 28, 279-321.

Pinkel, K., M. Merrifield and H. Ramm, 1995: Probing the interior of Arctic leads: investigations using high frequency sound. J. Geophys. Res., 100, 4693-4705.

Polonichko, V., 1997: Generation of Langmuir circulation in case of wind-wave misalignment. J. Geophys. Res., 102, 1573-1580.

Smith, J.A. and J. Largier, 1995: Observations of nearshore circulation: rip currents. J. Geophys. Res., 100, 10967-10975.

Thorpe, S.A., 1995: Dynamical processes of transfer at the sea surface. Progr. Oceanogr., 35 , 315-352.

Weller, R.A. and J.F. Price, 1988: Langmuir circulation within the oceanic mixed layer. DeepSea Res., 35, 711-747. 